\title{
Laser Structuring for Control of Coupling Between THz Light and Phonon Modes
}

\author{
Xuewen Wang, ${ }^{1, *}$, Gediminas Seniutinas ${ }^{1,2}$, Armandas Balčytis ${ }^{1,3}$, Irmantas Kašalynas ${ }^{3}$, Vytautas Jakštas ${ }^{3}$, Vytautas Janonis ${ }^{3}$, \\ Rimvydas Venckevičius ${ }^{3}$, Ričardas Buividas ${ }^{1}$, Dominique Appadoo ${ }^{4}$, Gintaras Valušis ${ }^{3}$ and Saulius Juodkazis ${ }^{1}$ \\ ${ }^{1}$ Centre for Micro-Photonics, Faculty of Engineering and Industrial Sciences, Swinburne University \\ of Technology, Hawthorn, VIC 3122, Australia \\ E-mail: xuewenwang@swin.edu.au \\ ${ }^{2}$ School of Mathematical and Physical Sciences, University of Technology Sydney, Thomas St, Ulti- \\ mo, NSW 2007, Australia \\ ${ }^{3}$ Center for Physical Sciences and Technology, Sauletekio al. 3, LT-10222 Vilnius, Lithuania \\ ${ }^{4}$ Australian Synchrotron, Blackburn Road, Clayton, Victoria 3168, Australia
}

\begin{abstract}
Modification of surface and volume of sapphire is shown to affect reflected and transmitted radiation at $\mathrm{THz}$ spectral range. Structural modifications were made using ultra-short 230 fs laser pulses at 1030 and $257.5 \mathrm{~nm}$ wavelengths forming surface ripples of $\sim 250 \mathrm{~nm}$ and $60 \mathrm{~nm}$ period, respectively. Softening of the transverse optical phonon $\mathrm{TO}_{1}$ mode due to disorder was most pronounced in reflection from laser ablated surface. It is shown that sub-surface periodic patterns of laser damage sites have also modified reflection spectra due to coupling of $\mathrm{THz}$ radiation with phonons. Application potential of laser structuring and disordering for phononic engineering is discussed.
\end{abstract}

DOI: $10.2961 /$ jlmn.2016.03.0017

Keywords: laser fabrication, nanotexturing, void formation, material engineering, phonon spectrum control, terahertz spectroscopy.

\section{Introduction}

Spectral properties at sub-1 mm wavelengths at around terahertz $\left(1 \mathrm{THz}=10^{12} \mathrm{~Hz}\right)$ frequencies are important for understanding weak interaction in peptides and proteins $[1,2]$, material response at vicinity of phase transitions $[3,4]$, glass formation where low frequency Raman spectra exhibit the low frequency $10-50 \mathrm{~cm}^{-1}$ boson peak due to rearrangement of density of states in amorphous materials [5]. In silk, amorphous and crystalline structural components with proteins forming a 3D network of random and $\alpha$-coils together with a crystalline $\beta$-sheet phase can be distinguished in the THz spectral window [6-11].

Control of phonon spectrum is a new frontier in material science for growth and deposition of layered structures of usually incompatible materials with different thermal expansion coefficients. Materials' optical properties at IR range can be engineered through control of the phonon spectrum. For example, surface phonon polaritons (SPP) are shown to control directionality of black body emission when coupled with surface gratings [12]. By introducing patterns with period $\Lambda$ satisfying the grating equation:

$$
\frac{2 \pi}{\lambda} \sin \theta=k_{\|}+m \frac{2 \pi}{\Lambda},
$$

where $\lambda$ is the wavelength of emitted light (black body radiation), $\theta$ is the angle of emitted light, $k_{\|}$is the surface wave component parallel to the surface (SPP), and $m$ is an integer. It is thereby possible to create a strong directional out-coupling of the IR emission into free space out of the sample [12]. With a surface grating $\Lambda=0.55 \lambda$ directional emission was observed at $\lambda=11.36 \mu \mathrm{m}$ from SiC surface with intensity 20 times larger as that from a flat surface at the same temperature [12]. This is called Wolf's effect: at the angle, $\theta$, of the largest emissivity, the reflectivity, $R$, has a dip. This corresponds to Kirchhoff's law: the polarized directional spectral emissivity $\varepsilon$ is equal to the absorptivity $\alpha$ and is given by $\varepsilon \equiv \alpha=1-R$.

Surface nanotexturing of sapphire by fs-laser ablation is shown to enhance light extraction efficiency in light emitting diodes [13]. Surface patterns with periods about 1$5 \mu \mathrm{m}$ with different duty cycles are used for epitaxial lateral overgrowth (ELO) for GaN to reduce the density of threading dislocations. A better understanding of nanotexturing effects at the T-ray spectral range (phonon modes) is required for future material engineering. Nano-/micro-scale surface modifications can be readily made by direct writing with ultra-short fs-laser ablation.

Here, laser modifications on/in sapphire are identified in reflection at the $\mathrm{THz}$ spectral range. Such nano-/microscale patterns can be used for controlling the phonon spectrum on the surface and in the bulk of polar semiconductors and dielectrics, hence, affecting heat transport, emissivity, coupling mechanisms between surface excitation in layered structures [14].

\section{Samples and procedures}

The THz/Far-IR Beamline at the Australian synchrotron was used to characterize laser modified samples at $40-$ $800 \mathrm{~cm}^{-1}$ spectral range. The beamline is equipped with a Bruker IFS 125/HR Fourier Transform (FT) spectrometer and Opus software was used for initial data analysis. Up to 
(a)

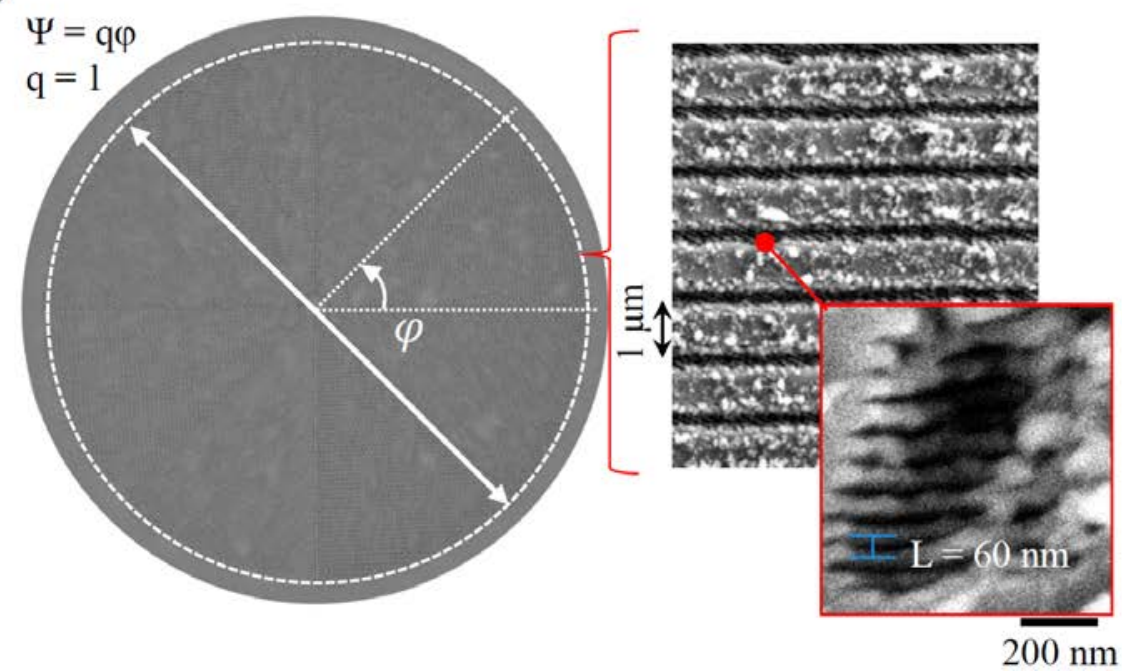

(b)

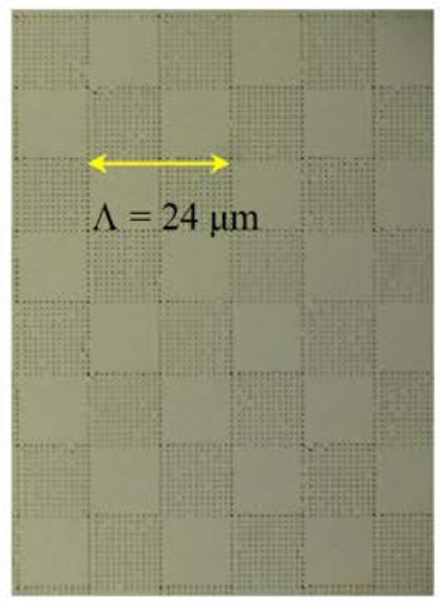

Fig. 1 (a) SEM images of a $q$-plate pattern recorded on sapphire at different magnifications. The azimuthal dependence of the slow axis is given by $\Psi=q \varphi$ where $q=1$ and $\varphi$ is the polar angle. Conditions: pulse energy $E_{\mathrm{p}}=4.25 \mathrm{~nJ}$ (on sample), $\lambda=257 \mathrm{~nm}$ wavelength, $\tau_{\mathrm{p}}=230 \mathrm{fs}$ pulse duration, $v=1 \mathrm{~mm} / \mathrm{s}$ scan speed, repetition rate $f=200 \mathrm{kHz}$, focusing with $N A=0.4$ objective lens (irradiance $I_{\mathrm{p}}=3.8 \mathrm{TW} / \mathrm{cm}^{2} /$ pulse). Diameter of $q$-plate is $200 \mu \mathrm{m}$ and fabrication time $4 \mathrm{~min}$. (b) Photo image of a grating inscribed in sapphire at 20 - $\mu \mathrm{m}$-depth by fs-laser $1030 \mathrm{~nm} / 230 \mathrm{fs}$ pulses using numerical aperture $N A=1.42$ objective lens at $v=1 \mathrm{~mm} / \mathrm{s}$, repetition rate $f=50 \mathrm{kHz}, E_{\mathrm{p}}=1.52 \mu \mathrm{J} /$ pulse.

100 spectral scans were captured and averaged to improve signal-to-noise $(\mathrm{S} / \mathrm{N})$ ratio. All measurements were conducted at room temperature. Also, the spectra of reflection and transmission at normal incidence were obtained for the range of 20-450 $\mathrm{cm}^{-1}$ with a resolution of $4 \mathrm{~cm}^{-1}$, using a customized Fourier-transform infrared (FT-IR) spectrometer with an evacuated chamber. The spectra were each measured 3 times at different sample positions, averaging up to 100 spectral scans. Variations between each of the spectra were within $10 \%$. In the $100-400 \mathrm{~cm}^{-1}$ spectral range the FT-IR spectrometer operates at $\sim 5 \%$ precision.

Samples of c-plane sapphire (Shinkosha Ltd.) were used for femtosecond (fs-)laser structuring at $\lambda=1030$ and $257.5 \mathrm{~nm}$ wavelengths (Pharos, Light Conversion). Fabrication conditions at $\lambda=257 \mathrm{~nm}$ : pulse duration $\tau_{\mathrm{p}} \sim 230 \mathrm{fs}$, repetition rate of $f=0.2 \mathrm{MHz}$, linear scan speed of $v=1 \mathrm{~mm} / \mathrm{s}$. Exposure pattern was controlled via an integrated software-hardware solution (Workshop of Photonics, Ltd) equipped with Aerotech stages. Focusing objective lens of numerical aperture $N A=0.4$ (50× magnification, PlanApo UV, Mitutoyo), which focused into focal spot with waist (radius) $w_{0}=0.61 \lambda / N A \approx 390 \mathrm{~nm}$. The number of pulses per focal spot was $N=2 w_{0} \times f / v=157$. Fabrication conditions at $\lambda=1030 \mathrm{~nm}$ were: $N A=1.42$ (100× magnification, PlanApo NIR, Mitutoyo) at $v=1 \mathrm{~mm} / \mathrm{s}, f=50 \mathrm{kHz}$, $E_{\mathrm{p}}=1.52 \mu \mathrm{J} /$ pulse. A single pulse irradiance would correspond to $I_{\mathrm{p}}=1.1 \mathrm{PW} / \mathrm{cm}^{2}$ and pulse power of $P_{\mathrm{p}}=6.6 \mathrm{MW}$, which is above the self-focusing threshold. Together with a spherical aberration due to a deep focal spot position this smears the pulse energy axially, however, it is above the intrinsic threshold of void formation $\sim 10 \mathrm{TW} / \mathrm{cm}^{2}$ [16]. In both cases, on the surface and in the bulk strong structural damage was induced.

\section{Results and discussion}

Figure 1(a) shows a pattern of grooves comprising an inhomogeneous birefringent plate with a topological defect of charge $q$ (hence $q$-plate) for the azimuthal patterning of the optical axis. The azimuthal dependence of the slow axis is given by $\Psi=q \varphi$, where, $q$ is the half integer and $\varphi$ is the polar angle. The laser ablated regions have a nanoscale pattern of ripples with periods scaling with wavelength and the refractive index, $n$, as $\Lambda=\lambda /(2 n)$ on dielectric (transparent material) surfaces. Hence, through variation of wavelength different nano-structures can be formed. At tight focusing below the surface, voids can be created inside dielectric host materials when a single pulse irradiance is $I_{\mathrm{p}}>10 \mathrm{TW} / \mathrm{cm}^{2}$ (Fig. 1(b)). Such surface and bulk modifications can be patterned with high precision and their use for tailoring optical, mechanical, and thermal properties are of interest. Optical characterization of such patterns at Tray region was carried out. Sapphire can be used as a substrate for optical elements due to its high transparency (Fig. 2) and performance of laser inscribed optical elements in Tray region has to be well understood.
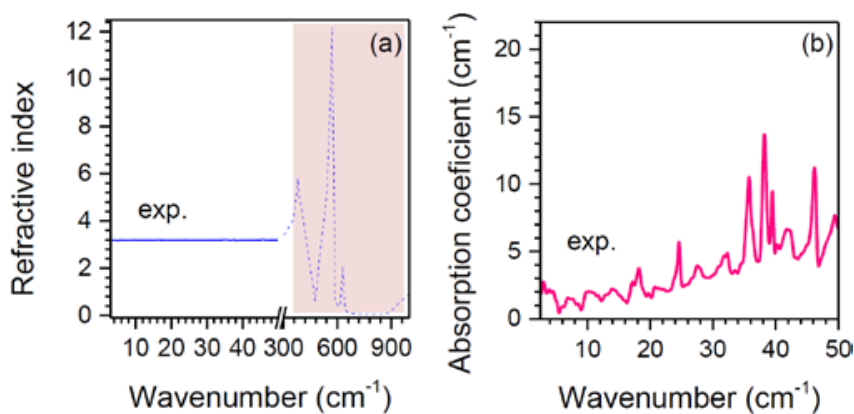

Fig. 2 Measured (exp.) refractive index, $n$, (a) and absorption coefficient, $\alpha_{\mathrm{s}}$, (b) of sapphire (c-face) in the T-ray region. Tabulated $n$ values [15] for higher THz range are added in (a). The refractive index and absorption coefficient of sample were measured using a THz-TDS system (Teravil-Ekspla).

The TO phonon modes in $\alpha-\mathrm{Al}_{2} \mathrm{O}_{3}$ are located at 385$388 \mathrm{~cm}^{-1} \quad\left(\mathrm{TO}_{1}\right), 439 \mathrm{~cm}^{-1}\left(\mathrm{TO}_{2}\right)$ and $483 \mathrm{~cm}^{-1}\left(\mathrm{LO}_{2}\right)$ branches, $569 \mathrm{~cm}^{-1}\left(\mathrm{TO}_{3}\right)$ and $630 \mathrm{~cm}^{-1}\left(\mathrm{LO}_{3}\right), 633 \mathrm{~cm}^{-1}$ $\left(\mathrm{TO}_{4}\right)$ and $1021 \mathrm{~cm}^{-1}\left(\mathrm{LO}_{4}\right)$ [17]; all the values were taken 
(a)
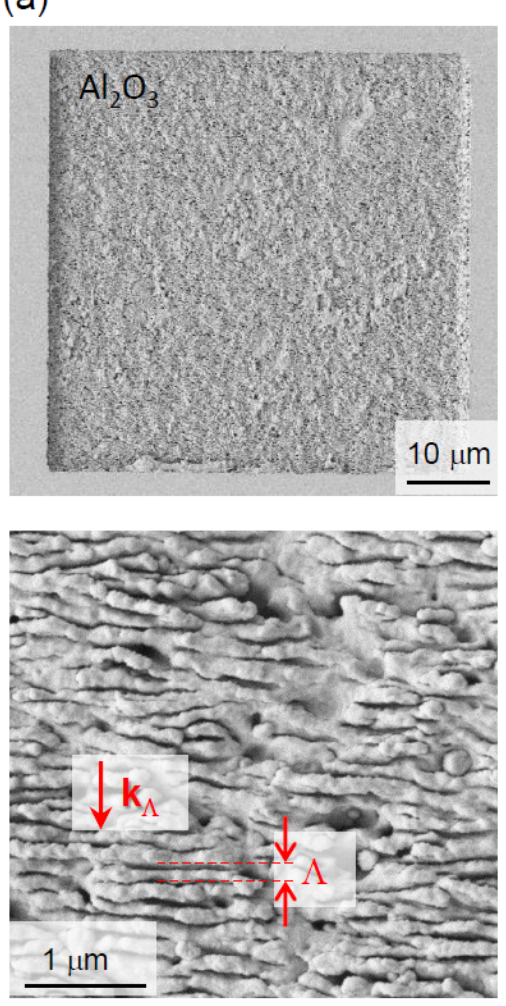
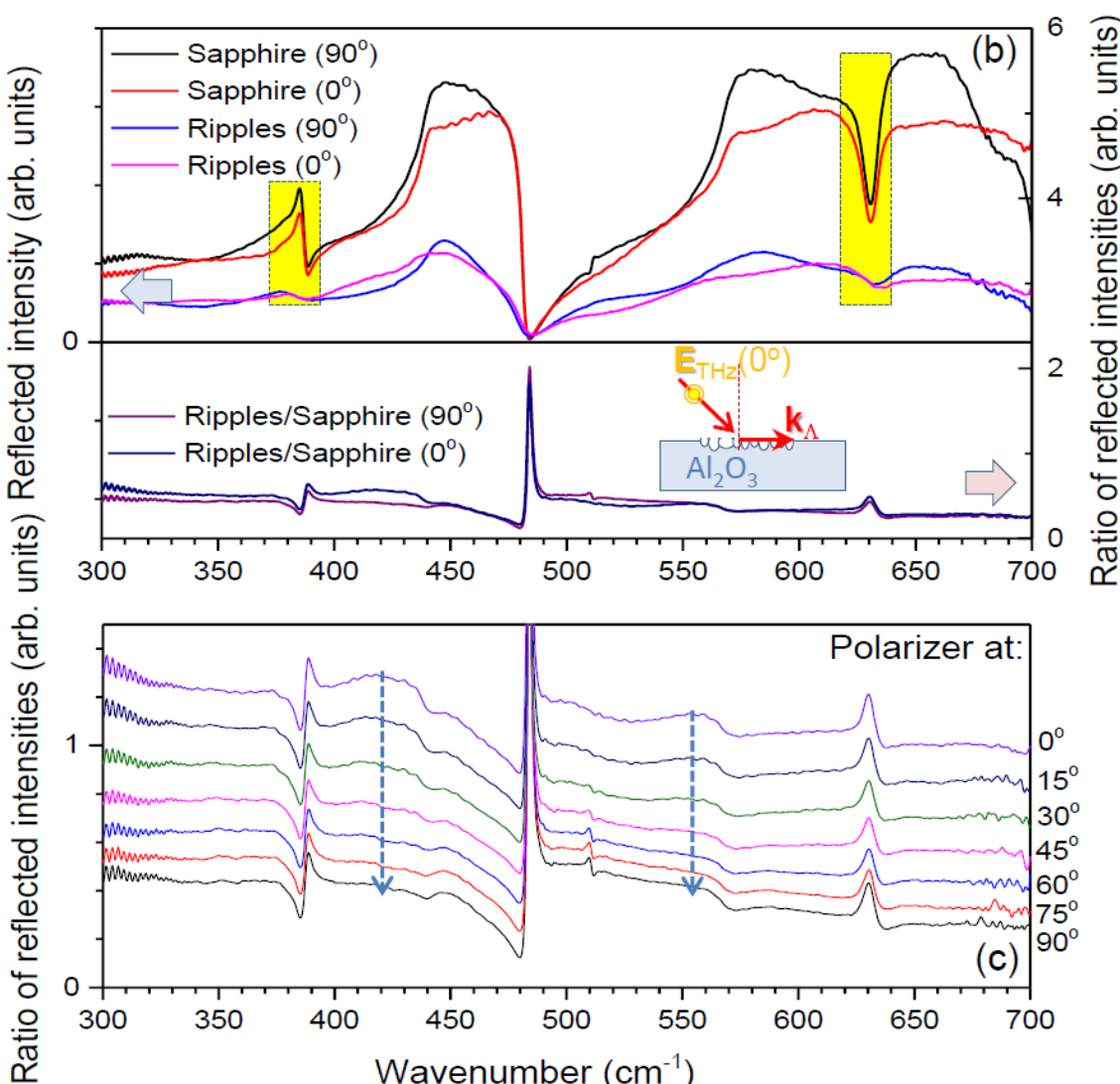

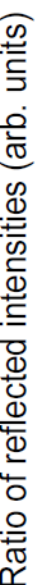

Fig. 3 (a) Typical SEM images of ripples laser ablated at $1030 \mathrm{~nm} / 280 \mathrm{fs}$ at different magnifications. Period of ripples $\Lambda$ whereas $\boldsymbol{k}_{\Lambda}$ is the wavevector. (b) Reflection from optical sapphire flat and ablated surface (ripples) at two polarizations $0^{\circ}$ and $90^{\circ}$; a metal wire grating polarizer placed before the sample was used with $0^{\circ}$-orientation corresponding to polarization "aligned” (inset) with the surface ripples (or $\mathbf{E}_{\mathrm{THz}} \perp \mathbf{k}_{\Lambda} ; k_{\Lambda}=2 \pi / \lambda ;$ ) angle of incidence is close to normal. The highlighted box regions show spectral locations of the strongest modifications at the sapphire's TO $385 \mathrm{~cm}^{-1}$ and $\mathrm{TO}_{4} 633 \mathrm{~cm}^{-1}$ phonon modes. (c) Relative reflectivity at several polarization angles. Spectra are offset for clarity. Inset shows geometry of experiment. T-ray source was the IR beamline at the Australian synchrotron.

from Table 5.2 of Ref. [17]. However, the best numerical fit of experimental reflectivity of sapphire was achieved for $\mathrm{LO}_{4}$ at $906 \mathrm{~cm}^{-1}$ (not shown here). Figure 3(b) shows a strong alteration of reflectivity from laser ablated pattern of ripples at the $\mathrm{TO}_{1}$ mode at two different polarizer orientations. Self-organized ripple structures with period of $\sim 250 \mathrm{~nm}$ (Fig. 3(a)) or $\Lambda_{\mathrm{r}} \approx \lambda_{\mathrm{l}} /(2 n)$ were recorded at the $\lambda_{\mathrm{l}}=1030 \mathrm{~nm}$ laser wavelength used, and where refractive index of sapphire is $n=1.7$. Strong relative reflectivity increase at the position of $\mathrm{LO}_{2}$ can be partially caused by a very low value of $R$ for the c-plane sapphire sample (small values of denominator used in normalization) which turn the overall minor mode softening by laser structuring appear as a large ratio. However, the value of this peak remained consistent throughout the measurements at all different polarizations, hence, it being a numerical artifact appears unlikely. There is an observable change in $R$ at the spectral location of $\mathrm{TO}_{4}$ mode. Reflection from the laser ablated surface shows spectrally broader $\mathrm{TO}_{1,4}$ bands (Fig. 3(b)), a sign of mode softening. Reflectivity interrogated at different linear polarizer angles showed a consistent change in $R$ values of the $\mathrm{TO}_{1,4}$ bands (Fig. 3(c)).

Figure 4 shows transmission and reflection spectra from the pattern of laser inscribed lines with period $\Lambda \approx 24 \mu \mathrm{m}$. In the reflectance spectrum a broad band around $\lambda_{\mathrm{R}}=294 \mathrm{~cm}^{-1}$ was observed (or $\lambda_{\mathrm{R}}=34 \mu \mathrm{m}$ in free space with refractive index $n=1$ ). The reflected wavelength is also the same which is effectively absorbed, hence, coupled into the structure/sample. The momentum conservation for the wavevectors of reflected light $\mathbf{k}_{\mathrm{R}}$, structure/sample mode $\mathbf{k}_{\mathrm{s}}$, (could be a surface wave, SPP, TO-mode), and a grating $\mathbf{k}_{\mathrm{g}}$, is given by $\mathbf{k}_{\mathrm{R}}=\mathbf{k}_{\mathrm{s}}-\mathbf{k}_{\mathrm{g}}$. This is the simplest grating mediated two wave mixing scenario. One can find the corresponding wavelength $\lambda_{\mathrm{s}}$ from the definition $k_{\mathrm{s}} \equiv 2 \pi /\left(\lambda_{\mathrm{s}} / n\right)$, at which the energy is deposited (absorbed) from the right triangle rule:

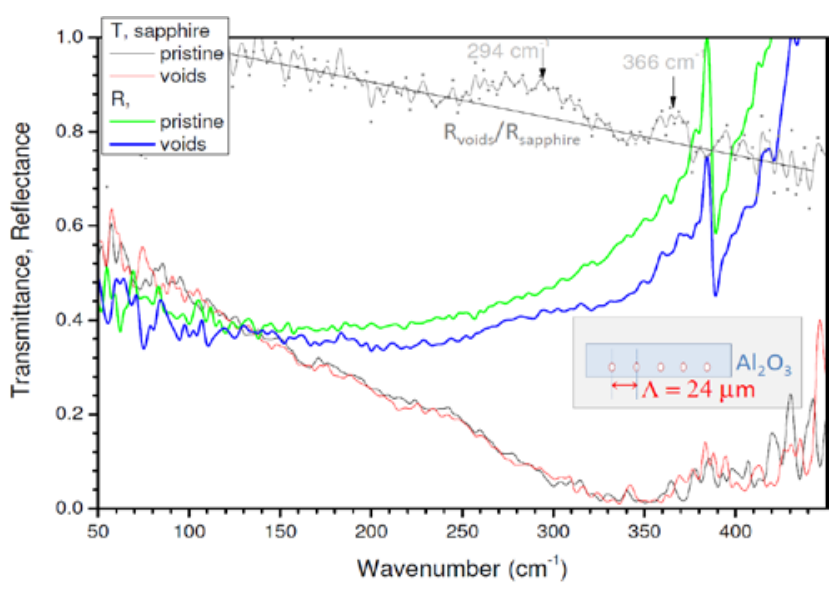

Fig. 4 Reflection and transmission spectra at normal incidence from $365-\mu \mathrm{m}$-thick two-side polished sapphire flats without and with void grating inscribed at $20 \mu \mathrm{m}$ depth. At wavenumbers larger than $400 \mathrm{~cm}^{-1}$ there are artifacts due to the low efficiency of the beam splitter used. Inset shows schematically a side view of the sample. 


$$
\left(\frac{2 \pi}{\lambda_{R} / n}\right)^{2}=\left(\frac{2 \pi}{\lambda_{s} / n}\right)^{2}-\left(\frac{2 \pi}{\Lambda}\right)^{2} .
$$

Equation 2 allows to calculate the wavelength and energy of the mode which efficiently absorbs incoming IR light and, hence, effectively reflects it. One would find $\lambda_{\mathrm{s}}=30.94 \mu \mathrm{m}\left(323 \mathrm{~cm}^{-1}\right)$ with $n=3.2$ in sapphire for the high transmission region (Fig. 2). This did not fit the $\mathrm{TO}_{1}$ mode. The second reflectivity peak at $366 \mathrm{~cm}^{-1}$ (or $\lambda_{\mathrm{R}}=27.3 \mu \mathrm{m}$ in free space) corresponds to the $\lambda_{\mathrm{s}}=25.64 \mu \mathrm{m}$ or $390 \mathrm{~cm}^{-1}$ (Eqn. 2), which match the $\mathrm{TO}_{1}$ [17]. Since a square lattice was inscribed in sapphire (Fig. 2(c)), there is a mode with period $\sqrt{2} \Lambda$. For this period the reflection peak at $366 \mathrm{~cm}^{-1}$ would correspond to $\lambda_{\mathrm{s}}=26.43 \mu \mathrm{m}$ or $378 \mathrm{~cm}^{-1}$, which is also close to the $\mathrm{TO}_{1}$. For the opaque region (shaded in Fig. $1(\mathrm{a})), n \approx(4.5-5.5)$ for the $\mathrm{TO}_{1,2}$ region and the same analysis using Eqn. 2 suggests the $366 \mathrm{~cm}^{-1}$ peak being close to the $\mathrm{TO}_{1}$ mode; similarly, the broad $294 \mathrm{~cm}^{-1}$ feature has no match with phonon mode. The broad $294 \mathrm{~cm}^{-1}$ reflection band can be due to a coupling with acoustic modes [14] and would require future experiments for different $\Lambda$ values matching the phonon modes.

Even though the reflectivity changes are very weak in the normalized spectrum (Fig. 4), they are discernable on a Rayleigh scattering slope (approximated by a line in this narrow spectral window). This proves that a designed periodic pattern can be used to couple energy into a specific phonon mode.

\section{Conclusions and outlook}

It is demonstrated that surface and volume structuring of sapphire has direct impact on softening TO phonon modes and changing reflectivity at specific modes. Laser induced damage and structural disorder is the reason of this modification of the phonon spectrum. Control over phonon spectrum is strongly required for the growth of layered structures where different optical and acoustic modes are coupling with the interface waves [14]. Among other possible applications are surface micro-texturing for ELO solutions in GaN-based LEDs, an electron mobility control in structures with strong phonon scattering, and in thermoelectrical materials for enhancement of difference between electron and phonon mean free path. Also a fast growing field of applications is in the design of flat optical elements using surface patterning [18] where fs-laser surface and bulk structuring has been well developed [19-21].

\section{Acknowledgments and Appendixes}

This study was carried out as a part of the Australiansynchrotron beamtime proposal M8468-2014. Partial support via Research Council Discovery grant DP130101205 and collaborationproject with Workshop of Photonics, Ltd. is highly appreciated. Polished sapphire samples were donated by Tecdia Ltd. Financial support from the Research Council of Lithuania under the KITKAS project, contract No.LAT-04/2016. We acknowledge Teravil-Eksplafor the access to a commercial T-Spec THz-TDS system.

\section{References}

[1] G. Mlnonl and G. Zerbl: J. Phys. Chem., 86, (1982) 4791.

[2] G. M. Png, R. J. Falconer and D. Abbott: IEEE Trans. Terahertz Sci. Technol., 6, (2016) 45.

[3] J. Doucet, I. Denicolo and A. Craievich: J. Chem. Phys., 75, (1981) 1523.

[4] J. D. Barnes and B. M. Fanconi: J. Chem. Phys., 56, (1972) 5190.

[5] A. I. Chumakov, G. Monaco, A. Monaco, W. A. Crichton, A. Bosak, R. Rüffer, A. Meyer, F. Kargl, L. Comez, D. Fioretto, H. Giefers, S. Roitsch, G. Wortmann, M. H. Manghnani, A. Hushur, Q. Williams, V. J. Balogh, V. K. Parlinski, P. Jochym, and P. Piekarz: Phys. Rev. Lett., 106, (2011) 225501.

[6] X. Liu and K.-Q. Zhang: "Silk Fiber - Molecular Formation Mechanism, Structure-Property Relationship and Advanced Applications” ed. by C. Lesieur (InTech, 2014) p.69.

[7] S. Kim, B. Marelli, M. A. Brenckle, A. N. Mitropoulos, E.-S. Gil, K. Tsioris, H. Tao, D. L. Kaplan and F. G. Omenetto: Nat. Nanotechnol., 9, (2014) 306.

[8] A. Balčytis, M. Ryu, G. Seniutinas, J. Juodkazytė, B. C. C. Cowie, P. R. Stoddart, J. Morikawa and S. Juodkazis: Nanoscale, 7, (2015) 18299.

[9] Y.-L. Sun, Q. Li, S.-M. Sun, J.-C. Huang, B.-Y. Zheng, Q.-D. Chen, Z.-Z. Shao and H.-B. Sun: Nat. Commun., 6, (2015) 8612.

[10] Y. Hu, Q. Zhang, R. You, L. Wang and M. Li: Adv. Mat. Sci. Engineer., 2012, (2012) 185905/1.

[11] J. Morikawa, M. Ryu, K. Maximova, A. Balčytis, G. Seniutinas, L. Fan, V. Mizeikis, J. L. Li, X. W. Wang, M. Zamengo, X. Wang and S. Juodkazis: RSC Adv., 6, (2015) 11863.

[12] J.-J. Greffet, R. Carminati, K. Joulain, J.-P. Mulet, S. Mainguy and Y. Chen: Nature, 416, (2002) 61.

[13]E. Jelmakas, A. Kadys, T. Malinauskas, D. Paipulas, D. Dobrovolskas, M. Dmukauskas, A. Selskis, S. Juodkazis and R. Tomašiūnas: J. Physics D: Appli. Phys., 48, (2015) 285104.

[14] Y. Ishitani: J. Appl. Phys., 112, (2012) 063531.

[15]E. R. Dobrovinskaya, L. A. Lytvynov and V. Pishchik: "Sapphire: Material, Manufacturing, Applications", (Springer US, New York, 2009).

[16] O. Efimov, S. Juodkazis and H. Misawa: Phys. Rev. A, 69, (2004) 042903.

[17] M. Schubert: "Infrared Ellipsometry on Semiconductor Layer Structures: Phonons, Plasmons, and Polaritons”, (Springer-Verlag Berlin Heidelberg, Berlin, 2004) p.75.

[18] J. JJ Nivas, S. He, A. Rubano, A. Vecchione, D. Paparo, L. Marrucci, R. Bruzzese and S. Amoruso: Sci. Rep., 5, (2015) 17929.

[19]E. Vanagas, I. Kudryashov, D. Tuzhilin, S. Juodkazis, S. Matsuo and H. Misawa: Appl. Phys. Lett., 82, (2003) 2901.

[20]S. Juodkazis, S. Matsuo, H. Misawa, V. Mizeikis, A. Marcinkevičius, H. B. Sun, Y. Tokuda, M. Takahashi, T. Yoko and J. Nishii: Appl. Surf. Sci., 197-198, (2002) 705.

[21] M. Malinauskas, A. Žukauskas, S. Hasegawa, Y. Hayasaki, V. Mizeikis, R. Buividas and S. Juodkazis: Light: Sci. Appl., 5, (2016) e16133.

(Received: May 22, 2016, Accepted: October 6, 2016) 